\title{
Nanoscale study of conduction through carbon nanotube networks
}

\author{
M. Stadermann, ${ }^{1, *}$ S. J. Papadakis, ${ }^{2}$ M. R. Falvo, ${ }^{3}$ J. Novak, ${ }^{4}$ E. Snow,${ }^{4}$ Q. Fu, ${ }^{5}$ J. Liu, ${ }^{5}$ Y. Fridman,${ }^{6}$ \\ J. J. Boland, ${ }^{7}$ R. Superfine, ${ }^{2,3,6}$ and S. Washburn ${ }^{2,3,6,8}$ \\ ${ }^{1}$ Department of Chemistry, University of North Carolina at Chapel Hill, Chapel Hill, North Carolina 27599, USA \\ ${ }^{2}$ Department of Physics and Astronomy, University of North Carolina at Chapel Hill, Chapel Hill, North Carolina 27599, USA \\ ${ }^{3}$ Curriculum in Applied and Materials Science, University of North Carolina at Chapel Hill, Chapel Hill, North Carolina 27599, USA \\ ${ }^{4}$ Naval Research Laboratory, Nanostructures Section Code 6876, Washington, DC 20375, USA \\ ${ }^{5}$ Gross Chemistry Laboratory, Duke University, Box 90354, Durham, North Carolina 27708, USA \\ ${ }^{6}$ Department of Computer Science, University of North Carolina at Chapel Hill, Chapel Hill, North Carolina 27599, USA \\ ${ }^{7}$ Department of Chemistry, Trinity College, Dublin 2, Ireland \\ ${ }^{8}$ Department of Biomedical Engineering, University of North Carolina at Chapel Hill, Chapel Hill, North Carolina 27599, USA
}

(Received 20 January 2004; published 18 May 2004)

\begin{abstract}
We present local conductance measurements of carbon nanotube networks with nanometer scale resolution and show that there are discrete drops in conductance that correspond to junctions of metallic nanotubes and semiconducting nanotubes. The anomalies of these networks compared to thin films are shown, and a new method of discerning between semiconducting and metallic single-wall carbon nanotubes is demonstrated.
\end{abstract}

DOI: $10.1103 /$ PhysRevB.69.201402

PACS number(s): 73.63.Fg, 73.63.Rt

\section{INTRODUCTION}

Carbon nanotube field effect transistors $(\mathrm{CNT}-\mathrm{FETs})^{1,2}$ have been shown to be very sensitive to ambient conditions: charge transfer due to gas adsorption to the nanotube ${ }^{3}$ or the contact ${ }^{4}$ can drastically change the conductance of the device. This makes CNT-FETs ideal systems for molecular sensing. It has been shown that coating the nanotubes with polymers can increase their selectivity in sensor applications, ${ }^{5}$ and it is hoped that further functionalization will provide sensitivity to specific compounds, such as proteins or sugars, or even larger objects, such as viruses.

There are significant challenges associated with fabrication of devices out of individual CNT. Placement is difficult, and CNTs display a wide variety of transport properties depending on their diameter and chirality. Random networks of carbon nanotubes have been explored as an alternative. ${ }^{6,7}$ Nanotube networks take up more space than individual single wall carbon nanotube (SWNT), but they are much easier to fabricate and show great promise for simple mass fabrication. The macroscopic behavior of these networks has been investigated in Ref. 6. Although it might appear that these networks could be readily treated by percolation theory, ${ }^{8}$ in practice the mat sizes for device applications have too few elements for this kind of analysis to be instructive. In this paper, we perform a nanoscale investigation of the current paths through nanotube networks. We find that the conductance drops sharply at metallic/semiconducting nanotube junctions, which we identify by backgating the sample. We also find that the most important parameter determining the local conductance at a certain point in the network is the number of connections at that point.

\section{DESIGN AND IMPLEMENTATION}

Two different types of nanotube samples were used. The first sample consisted of a silicon dioxide surface, upon which $20 \mathrm{~nm}$ of gold were deposited by sputtering. The gold is patterned by scratching. Then, tubes synthesized by laserablation suspended in 1-Methyl-pyrrolidine were spun onto the surface. This sample type provides networks with low interconnectivity. The second type of sample used nanotubes grown directly onto silicon dioxide substrates using chemical vapor deposition. ${ }^{9}$ Gold was deposited onto the surface by thermal evaporation and patterned by shadow-masking. On samples with back gates, aluminum was deposited on the back before the gold deposition, and then the sample was sintered for $30 \mathrm{~min}$ at $400^{\circ} \mathrm{C}$. The oxide thickness was $1 \mu \mathrm{m}$.

A conductance imaging microscope ${ }^{10}$ was used to take high-resolution conductance measurements on the network. This AFM method uses a tuning fork force probe. A solid $\mathrm{Pt} / \mathrm{Ir}$ tip is attached, which allows for simultaneous measurement of topography and conductance. A lock-in amplifier was used to process the current signal. The oscillation amplitude of the tuning fork is about $40 \mathrm{~nm}$ in all measurements, and scanning is performed with a frequency shift $\Delta f=2.3 \mathrm{~Hz}$. The sensors have a resonance frequency of $f_{0}$ $=12.5 \mathrm{kHz}$. The force between tip and sample was estimated from frequency shift/distance curves to be on the order of $80 \mathrm{nN}$. A voltage of $0.1 \mathrm{~V}$ was applied to the sample, while the tip remained grounded, unless noted otherwise. On samples with two contacts, the voltage is applied to one contact, and the other contact is unconnected.

\section{RESULTS AND DISCUSSION}

The behavior of current flow through SWNT networks differs from that through conductive thin films. The conductance does not decrease linearly with the distance from the electrode. Instead, we find for nanotube networks with poor interconnections, that the conductance through the network stays at the same order of magnitude throughout large parts of the network, but then decreases dramatically at certain points in the network, as shown in Fig. 1. The resistance increase at these points is on the order of $10^{8} \Omega$, and they 


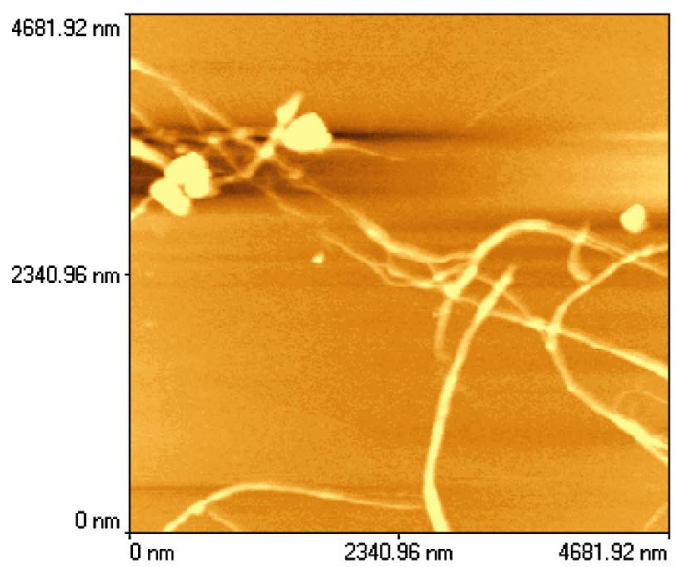

(a)

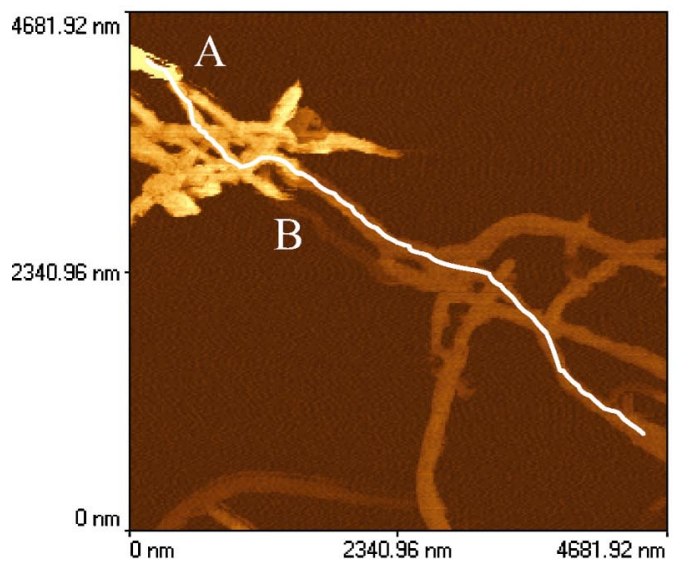

(b)
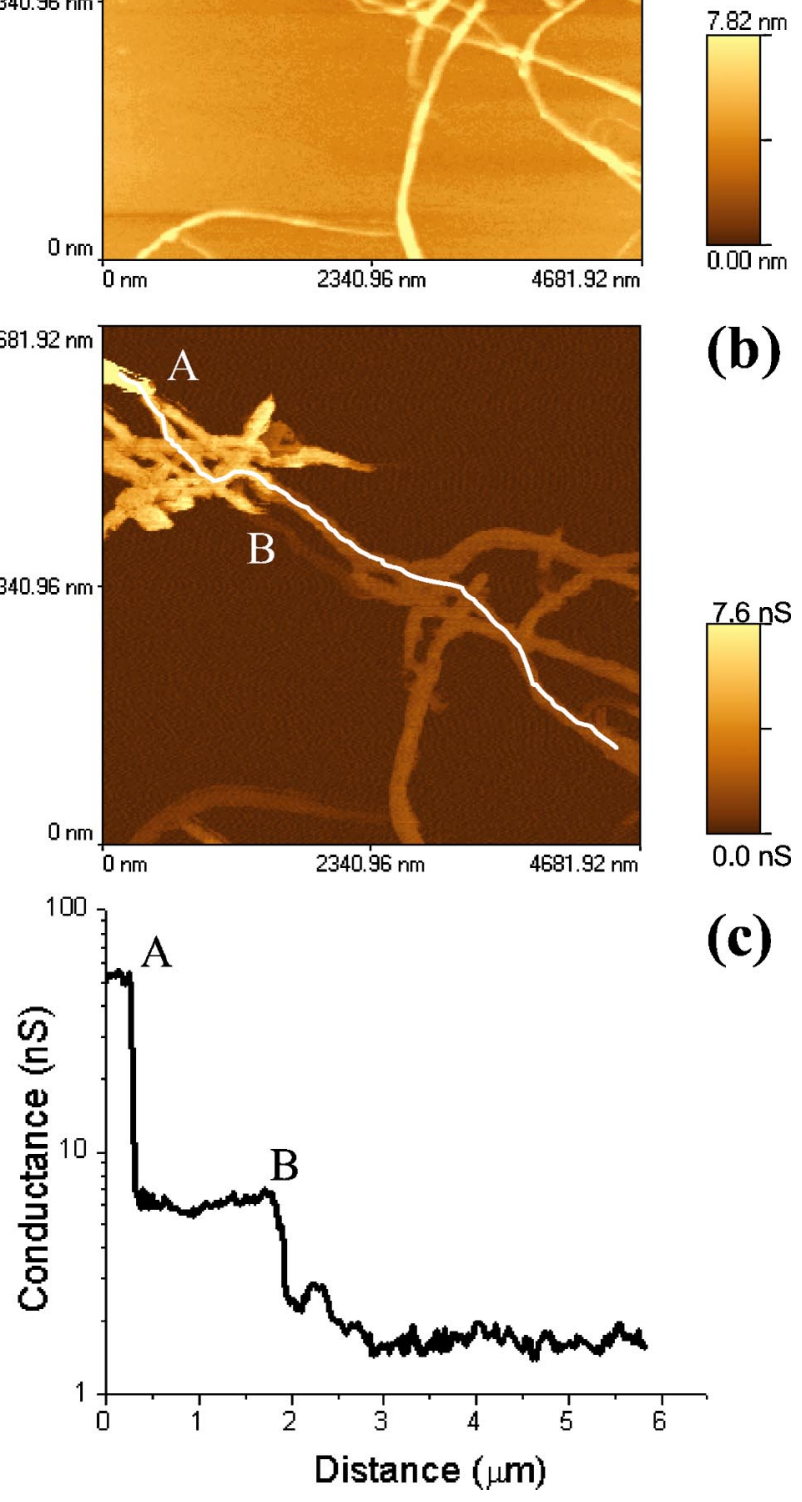

(c)

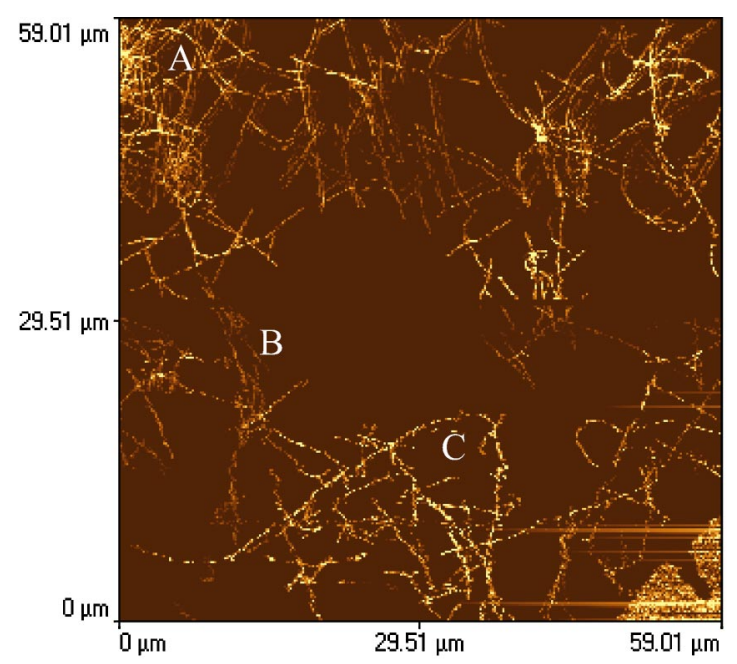

FIG. 2. (Color online) Conductance image of a nanotube network with high interconnectivity. These tubes were directly grown by CVD onto the sample. The electrode is in the bottom right corner. The conductances at points $A, B$, and $C$ are 700, 200, and $700 \mathrm{nS}$.
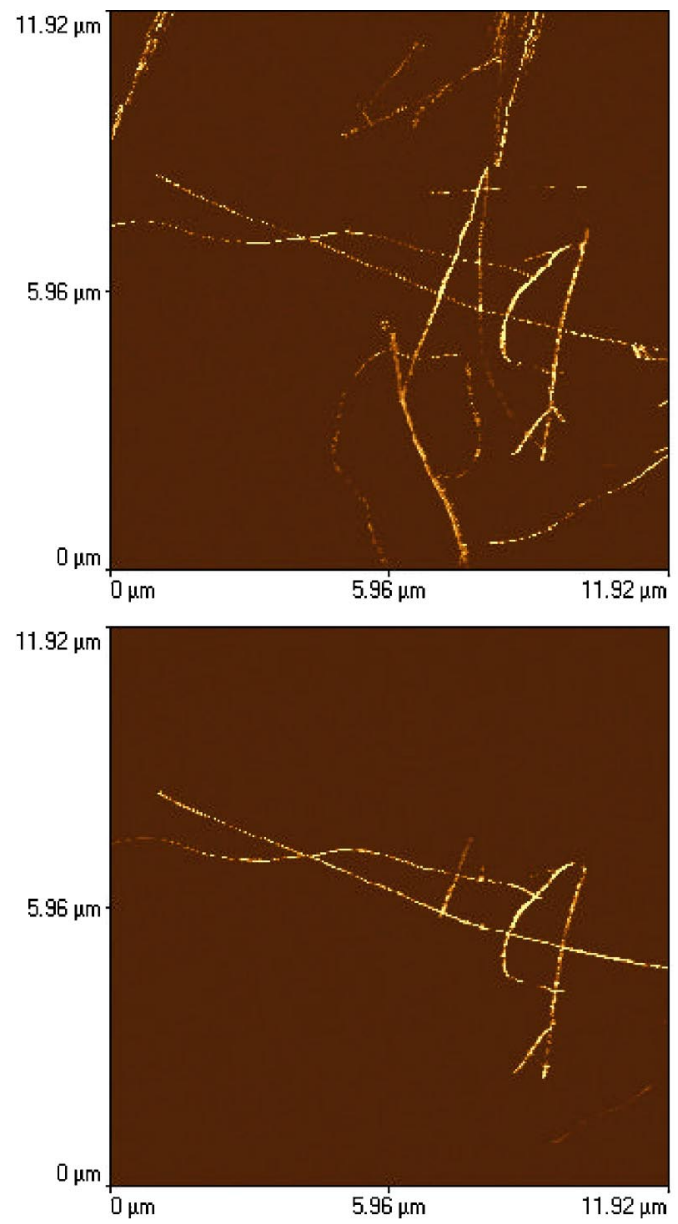

(b)

FIG. 1. (Color online) (a) Topography image of a nanotube network with low interconnectivity. The network contains bundles as well as individual tubes. These tubes were deposited from suspension and form clusters. (b) Conductance image of the same network taken simultaneously. The tubes appear to be thicker in this image than in (a). This effect only occurs on insulating substrates and may be related to charging. (c) Trace through the network, as indicated by the white line in (b). The electrode is at the top left (not shown). Conductance decreases coincide with nanotube junctions.

coincide with nanotube junctions. We believe that the conductance drops are associated with junctions formed by crossed semiconducting and metallic nanotubes, since this type of junction is a Schottky barrier ${ }^{11}$ and is known to have a significantly higher resistance than metallic/metallic or

FIG. 3. (Color online) A back gate voltage blocks current flow through semiconducting tubes. (a) Conductance image taken at $\mathrm{V}_{g}$ $=0 \mathrm{~V}$. (b) Conductance image taken at $\mathrm{V}_{g}=10 \mathrm{~V}$. Parts of the network visible in (a) disappear because they are connected through semiconducting tubes. 
semiconducting/semiconducting junctions.

In networks with high interconnectivity, we again find that the conductance does not decrease monotonically with increased distance from the electrode (Fig. 2). Instead, we can identify areas of high conductance more than $80 \mu \mathrm{m}$ away from the electrode. Local conductance is more dependent on the number of connections to the specific area: clusters of nanotubes with many paths to the electrode have significantly higher conductances than those parts of the network connected to the rest through fewer paths. Not only do multiple paths reduce resistance through the presence of multiple parallel resistors, but they also increase the probability that there is a chain of nanotubes stretching to the electrode that is purely metallic. Areas with low conductance typically only have two to three connections to the network, thus it is likely that these connections are dominated by the presence of highly resistive metallic/semiconducting junctions.

When a sufficient back gate voltage is applied to the sample, current flow through the semiconducting tubes in the network is suppressed (Fig. 3). This behavior is consistent with earlier work on individual SWNT field effect transistors. The bands in the semiconducting tube are shifted downwards in energy by a positive applied voltage, reducing the number of carriers in the tube, and, more importantly, increasing the width of the Schottky barrier at the contact that dominates the transport through the tube. ${ }^{12}$ The semiconducting tubes and all parts of the network connected by semiconducting tubes are no longer visible in the conductance image. Using this technique, we view only the purely metallic parts of the network and identify their spatial extent. Our data demonstrate that the majority of high-conductance connections consist of metallic tubes. When connecting the two

*Electronic address: staderma@email.unc.edu

${ }^{1}$ S. Tans, A.R.M. Verschueren, and C. Dekker, Nature (London) 393, 49 (1998).

${ }^{2}$ R. Martel, T. Schmidt, H.R. Shea, T. Hertel, and P. Avouris, Appl. Phys. Lett. 73, 2447 (1998).

${ }^{3}$ J. Kong, N. Franklin, C. Zhou, M. Chapline, S. Peng, K. Cho, and H. Dai, Science 287, 622 (2000).

${ }^{4}$ S. Heinze, J. Tersoff, R. Martel, V. Derycke, J. Appenzeller, and P. Avouris, Phys. Rev. Lett. 89, 106801 (2002).

${ }^{5}$ P. Qi, O. Vermesh, M. Grecu, A. Javey, Q. Wang, H. Dai, S. Peng, and K. Cho, Nano Lett. 3, 347 (2003).

${ }^{6}$ E. Snow, J. Novak, P. Campbell, and D. Park, Appl. Phys. Lett. electrodes on the sample macroscopically and measuring the change in source-drain current with applied gate voltage, we find for the sample in Fig. 3, at a source-drain voltage of $V_{s d}=100 \mathrm{mV}$ applied between the two electrodes, a current of $I_{s d}=2.94 \times 10^{-7} \mathrm{~A}$ at a gate voltage $V_{g}=0 \mathrm{~V}$, and $I_{s d}$ $=2.54 \times 10^{-7} \mathrm{~A}$ at $V_{g}=10 \mathrm{~V}$. Other samples show conductance changes between $1 \%$ and $50 \%$. This means that, in a mat of SWNT between two electrodes, a large part of the current is carried by a small number of highly conductive metallic connections, and the connections involving semiconducting tubes, even though much more numerous, carry a comparable or smaller portion of the current.

\section{CONCLUSION}

We have applied a new method for characterizing electrical networks to perform an analysis of current paths through a nanotube network. It was found that in networks with low interconnectivity, the conductance decreases in large steps of $\mathrm{G} \Omega$ magnitude with increasing distance from the electrode. In networks with high interconnectivity, the local conductance depends strongly on the number of connections to the rest of the network, and much less on distance to the electrode. We have demonstrated a new method for distinguishing between metallic and semiconducting SWNT, and used this method to determine the extent of purely metallic chains in a network.

\section{ACKNOWLEDGMENT}

This work was supported by the ONR-MURI program, and the Science Foundation Ireland.

82, 2145 (2003).

${ }^{7}$ J. Novak, E. Snow, E. Houser, D.P. and J.L. Stepnowski, and R. McGill, Appl. Phys. Lett. 83, 4026 (2003).

${ }^{8}$ A. Miller and E. Abrahams, Phys. Rev. 120, 745 (1960).

${ }^{9}$ Q. Fu, C. Lu, and J. Liu, Nano Lett. 2, 329 (2002).

${ }^{10}$ M. Stadermann, H. Grube, J. Boland, S. Papadakis, M. Falvo, R. Superfine, and S. Washburn, Rev. Sci. Instrum. 74, 3653 (2003).

${ }^{11}$ Z. Yao, H.W.C. Postma, L. Balents, and C. Dekker, Nature (London) 402, 273 (1999).

${ }^{12}$ J. Appenzeller, J. Knoch, V. Derycke, R. Martel, S. Wind, and P. Avouris, Phys. Rev. Lett. 89, 126801 (2002). 